\title{
OPTIMAL INSTALLATION OF SEVERAL RELATED EQUIPMENT WITH CONVERSION POSSIBILITY
}

\author{
Chan Onn Fong \\ University of Malaya
}

(Received September 4, 1978)

\begin{abstract}
The problem in which a firm has to meet the demand for the services of several distinct but related equipment over a planning horizon is considered. An equipment of one type can be converted into an equipment of the other type at some costs. Hence demands may be met by direct capacity installation (expansion) or by conversion from another type of equipment. Capacity installation and conversion costs are assumed to be concave reflecting possible economies of scale in these activities. The objective is to find a policy of capacity installations and conversions between the types of equipment such that the present value of the total installation and conversion costs is minimized. The problem is formulated and given a network representation. A dynamic programming algorithm, an extension and refinement to that developed in [2], is then developed which can be used to solve the problem efficiently when the number of distinct equipment is not too large.
\end{abstract}

\section{Introduction}

The problem in which a firm has to meet the demand for two types of services with two types of equipment available, an expensive general-purpose equipment and a cheaper specialized equipment which could provide only one of the services, has been studied (but not solved) in [6]. The objective of the problem is to determine the policy which minimizes the present value of installation (expansion) costs over an infinite horizon. In [2], it is shown that a discrete time version of this problem can be solved efficiently using a dynamic programming algorithm based on a derived recursive relation. An important generalization of this problem of capacity expansion with specialization is the case where a firm has to meet the demand for the services of several distinct but related equipment. An equipment of one type can be converted into an equipment of another type at some costs. Practical situations in which this problem of capacity installation with conversion 
possibility is applicable are many and can be found especially in the areas of transportation, manufacturing and communications. An example is the case of a pub1ic railway administration which has to meet the demand for passenger and freight services over a planning horizon. A passenger car can be converted into a freight car at some costs and vice versa. The objective of the organization is to determine a policy of capacity installations and conversions such that the discounted installation and conversion costs are minimized over the planning horizon. Another example is the case of a firm which uses a number of related manufacturing processes. The machinery of one process can be converted into the machinery of another process with some adjustment and rearrangement. Its objective is again to determine a policy of capacity installations and (if necessary) conversions such that the total discounted cost is minimized. In this paper, we formulate the capacity installation with conversion possibility problem into a finite discrete time mode1 with concave installation and conversion cost functions reflecting fixed costs and economies of scale in these activities. Although the problem so formulated is quite different from the inventory models of [10], [11], [12] and the one region and the two region capacity expansion model considered in [7] and [2] respectively, we show that an extension of the approaches used in these references (in particular that of [2]) can be used to solve the problem efficiently.

In the next section we provide a statement and formulation of the problem. A graphic representation of the problem and some properties of the extreme points are given in §3. The derived extreme point properties are utilized in \$4 to derive an efficient dynamic programming algorithm to solve the problem. A numerical example illustrating the application of the algorithm is given in $\$ 5$. In the last section we show how the problem can be extended to incorporate initial capacities, backlogging (or short-term leasing) of capacities, and arbitrary and not necessarily non-decreasing demands .

\section{Problem Formulation}

\subsection{Statement of problem}

The problem considered in this paper can be stated formally as follows: A firm has to satisfy the demand for the service of each of a number of equipment over a discrete finite time horizon. The demand for each equipment service is a known non-decreasing function of time. It must also be satisfied 
exactly (i.e. no inventory or backlogging of the services are allowed) at the end of each period by direct capacity installation or by conversion from the other type of equipment. It should be pointed out that for service industries (e.g. the transport industry) to which this mode1 is particularly applicable, this assumption is not restrictive since it is not possible to backlog or hold as inventory the services provided by the equipment [9, pp. 329]. The initial capacity of each equipment is assumed zero and capacity may be installed or converted at the beginning of each period. It is assumed that a unit of capacity installed or converted in any time period has a unit service capability until the end of the time horizon. Capacity installation and conversion costs are assumed to be concave, reflecting possible fixed costs and economies of scale in these activities. The problem is to find a policy of capacity installations and conversions between the different types of equipment such that the present value of the total installation and conversion costs is minimized.

Although the focus of this paper is on the problem so stated, in $\S 6$ we discuss some important extensions to the problem (e.g. when there are initial capacities or when the demands are not necessarily non-decreasing) and show how these could be handled as well.

\subsection{Mathematical formulation}

We begin the formulation process by defining

$$
\begin{aligned}
J= & \{1,2, \ldots n\}, \text { the set of types of equipment where } n \text { is } \\
& \text { the number of equipment type. } \\
J_{i}= & J-\{i\} \text { where } i \varepsilon J . \\
K= & \{1,2, \ldots T\}, \text { the set of time periods where } T \text { is the } \\
& \text { end of the planning horizon. }
\end{aligned}
$$

For $i \varepsilon J, t \varepsilon K$ define

$r_{i t}=$ the known increment in demand for type $i$ equipment during period $t$. Demand is non-decreasing, so $r_{i t} \geqslant 0$. Further, demand for each type of equipment is assumed to be measured in the same units.

$x_{i t}=$ the amount of capacity of type $i$ equipment installed in period $t$. Since capacity levels can only increase we have $x_{i t} \geqslant 0$.

$y_{i j t}=$ the amount of capacity of type $i$ equipment converted to type $j(j \neq i)$ in period $t$. Obviously we have $y_{i j t} \geqslant 0$. 
$I_{i t}=$ idle or excess capacity in type $i$ equipment in period $t$. In this formulation, for brevity, we do not permit backlogging (or short-term leasing) of capacity and hence $I_{i t} \geqslant 0$. In $\$ 6$ we show how this assumption can be relaxed. $c_{i t}\left(x_{i t}\right)=$ the cost of installing $x_{i t}$ units of type $i$ equipment $i_{r_{h}}$ time $t$.

${ }_{i j t}\left(y_{i j t}\right)=$ the cost of converting $y_{i j t}$ units of type $i$ equipment to type $j(j \neq 1)$ in time $t$.

The functions $C_{i t}(\cdot)$ and $H_{i j t}(\cdot)$ are assumed to be concave, nonnegative and non-decreasing in the interval $[0, \alpha)$, and are further assumed to be expressed in present value form. We also assumed that the cost of maintaining idle capacity is negligible and can be ignored. For notational convenience we let

$$
R_{i}(s, t)=\sum_{\tau=s}^{t} r_{i \tau} \text { and } R(s, t)=\sum_{i \varepsilon J} R_{i}(s, t) .
$$

The problem of capacity installation and conversion can now be formulated as problem $P$ :

Minimize

$$
\sum_{t \varepsilon K} \sum_{i \varepsilon J} C_{i t}\left(x_{i t}\right)+\sum_{t \in K} \sum_{i \varepsilon J}\left\{\sum_{j \varepsilon J} H_{i j t}\left(y_{i j t}\right)\right\}
$$

subject to constraints

$$
\begin{gathered}
x_{i t}-\sum_{j \varepsilon J} y_{i j t}+\sum_{j \varepsilon J} y_{j i t}+I_{i t-1}-I_{i t}=r_{i t} \text { for } t \varepsilon K, i \varepsilon J \\
I_{i 0}=0 \text { for } i \varepsilon J \\
I_{i T}=0 \text { for } i \varepsilon J \\
x_{i t}, y_{i t}, I_{i t} \geqslant 0 \text { for } i \varepsilon J, t \varepsilon K .
\end{gathered}
$$

Objective function (1) determines the minimum total present value of capacity installation and conversion costs. Constraint (2) represents the capacity balance equation of type $i$ equipment at each time period. It expresses the condition that the change in excess capacity level of type $i$ equipment from period $t-1$ to $t$ (i.e. $I_{i t}-I_{i t-1}$ ) is equal to the capacity increment $x_{i t}$ plus capacity converted into type $i$ equipment from other types of equipment (i.e. $\sum_{j \varepsilon_{i}} y_{j i t}$ ) less the capacity converted into the other types of equipment (i.e. $\sum_{j \varepsilon J} y_{i j t}$ ) and demand increment $r_{i t}$. Constraints (3) and (4) reflect the initial and terminal conditions respectively of the capacity states. 
Let $G$ represents the constraint set (2) - (5). $G$ is a closed convex set bounded from below and the cost functions are defined such that $P$ has a finite minimum. It is we11-known [4] that an optimal solution of a concave function over a closed convex set occurs at an extreme point of the constraint set. Since objective function ( 1 ) is a concave function and $P$ has a finite minimum, an optimal solution to problem $P$, therefore, occurs at an extreme point of $G$. We next derive some properties of extreme points of $G$ which can be used to develop an efficient dynamic programming algorithm for solving the problem.

\section{Properties of Extreme Point}

\subsection{Graphic representation}

It can be easily be verified that $G$ has the structure of a node-arc incidence matrix which gives rise to the graphic representation as presented in Figure 1, for the case where $n=2$ and $T=3$.

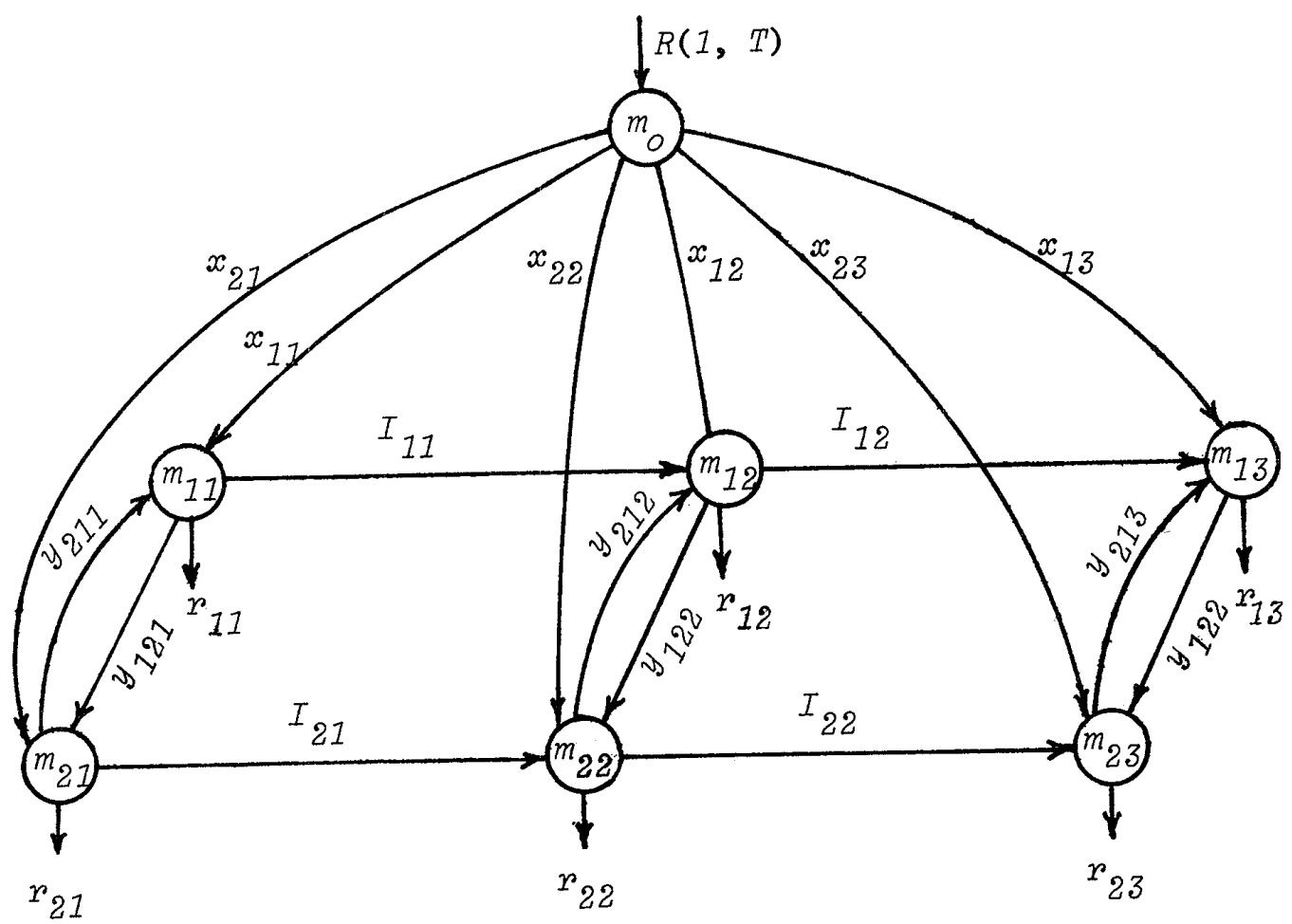

Figure 1: Graphic Representation for $P$ with $n=2$ and $T=3$. 
Each constraint in (2) is represented by flows through a node $m_{i t}$. The node $m_{0}$ represents the redundant equation obtained by summing up (2) over $t \varepsilon K$ and $i \varepsilon J$. Following the terminology of [2], we shall call an arc active if the flow along it is positive. An arc that is not active (i.e. have zero flow) is called inactive. An extreme flow (extreme point) of $G$ is defined here as a feasible flow whose active arcs contain no cycle [1], [5] implying that there exists an optimal solution to $P$ whose basic variables (active arcs) do not contain a cycle.

The graph representing $G$ is a single-source multi-sink network. From the results of [12] we can derive the following important properties to an extreme point of $P$. For completeness we also provide a simple proof for the properties.

Lemma 1. Every extreme point of $G$ satisfies

$$
\begin{aligned}
x_{i t} \cdot I_{i t-1}=0 & \text { for } i \varepsilon J, t \varepsilon K \\
x_{i t} \cdot y_{j i t}=0 & \text { for } i \neq j ; i, j \varepsilon j, t \varepsilon K \\
y_{j i t} \cdot I_{i t-1}=0 & \text { for } i \neq j ; i, j \varepsilon J, t \varepsilon K \\
y_{j i t} \cdot y_{k i t}=0 & \text { for } j \neq k ; i, j, k \varepsilon J, t \varepsilon K
\end{aligned}
$$

Proof: Consider (7a). $I_{i, t-1}>0$ implies that there exists a path of active arcs linking node $m_{i t-1}$ (via some nodes $m_{j \tau}, \tau \varepsilon\{1, \ldots, t-1\}$, $j \in J)$ to the only source node $m_{0}$. If $x_{i t}>0$ then we have traced a cycle of active arcs which include arcs $\left(m_{i t-1}, m_{i t}\right)$ and $\left(m_{0}, m_{i t}\right)$. This proves that both $x_{i t}$ and $I_{i, t-1}$ cannot be positive in an extreme point of $G$. Properties ( $7 \mathrm{~b})$ to ( $7 \mathrm{~d})$ can similarly be proved.

The graph $G$ representing problem $P$ is different from the network $V$ representing the problem considered in [2]. One major difference is that the network $V$ of [2] is a multi-source multi-sink network, whereas the graph $G$ of problem $P$ is a single-source multi-sink network. It is this difference which enables the properties of Lemma 1 to be developed for problem $P$, whereas no properties similar to Lemma 1 can be derived for the problem considered in [2]. Another major difference is that graph $G$ is applicable for problem $P$ with any number of types of equipment, whereas the network $V$ of [2] is generally applicable only when the number of regions is two. ${ }^{1}$

\footnotetext{
${ }^{1}$ The network $V$ of [2] can be extended to handle more than two producing regions, but only under the restrictive assumption that the transport cost into a region is independent of the supply source (see $\$ 5.6$ of [2]).
} 
We can characterize an extreme point of $G$ by defining $n+1$ groups of states at each period $t$ for $t \varepsilon K+\{0\}$ :

$$
\begin{aligned}
& \text { Group } 0: I_{i t}=0 \text { for } i \varepsilon J . \\
& \text { Group } i \text { for } i \varepsilon J: I_{i t}=0, I_{j t} \geqslant 0 \text { for } j \varepsilon J_{i} .
\end{aligned}
$$

Group 0 contains only one state. Any state of Group $i$ for $i \varepsilon J$ can be described by a $(n-1)$-dimensional variable. Further, the maximum feasible value of $I_{i t}$ in a state of group $i$ at time $t$ is $R(t+1, T)$. This can be easily verified from Figure 1. Representation of the possible levels of $I_{i t}$, when at most only $n-1$ of them can be.positive, by states of groups 0 , and $i$ for $i \varepsilon J$ provides a useful way of developing a dynamic programming algorithm which is discussed next.

\section{A Dynamic Programming Algorithm}

\subsection{Recursive relations}

States of groups 0 and $i$ for $i \varepsilon J$, however, do not completely exhaust all the possibilities since extreme points of $G$ can also satisfy

$$
\underset{i \varepsilon J^{\prime}}{\pi} I_{i t} \neq 0 \text { for } t \varepsilon K-\{T\} \text {. }
$$

Equation (7) is comparable, though obviously not similar, to (16) of [2] and a recursive relationship similar to (17) and (18) of [2] can be developed to solve problem $P$. For clarity, we shall develop the recursive relationship for solving problem $P$ here, and then point out the differences between the developed relationship and equations (17) and (18) of [2].

In the dynamic programming framework, let the time periods be stages and $\alpha$ be the $(n-1)$-dimensional state variable corresponding to the capacity levels associated with the states of group 0 , or $i$ for $i \varepsilon J$. From $\$ 3.1$ we know that at period $t$ group 0 has only one value while group $i$ has $(R(t+1, T)+1)^{n-1}$ levels each. Thus at each $t$ there are $P_{t}\left\{=n(R(t+1, T)+1)^{n-1}+1\right\}$ possible values for $\alpha$, with each value of $\alpha$ corresponding uniquely to a value of: $I_{j t}$ for $j \varepsilon J_{i}$ with $I_{i t}=0$.

Let $f_{t}(\alpha)$ be the cost of optimal capacity installation and conversion for periods $t+1, \ldots, T$, with $I_{j t}$ for $j \in J_{i}$ (with $I_{i t}=0$ ) uniquely specified by $\alpha \varepsilon\left\{1, \ldots, P_{t}\right\}$. Let $c_{u v}(\alpha, \beta)$ denote the cost of following an optimal policy over periods $u+1$ to $v$ given that $I_{i u}$ for $i \varepsilon J$ and $I_{i v}$ for $i \varepsilon J$ are specified by $\alpha$ and $\beta$ respectively and $\pi_{i \varepsilon J} I_{i t} \neq 0$ for $t=u+1, \ldots, v-1$. We have: 


$$
\begin{gathered}
f_{T}(\alpha=1)=0 \\
f_{u}(\alpha)=\operatorname{Min}_{u<v \leqslant T}\left\{\operatorname{Min}_{1 \leqslant \beta \leqslant P}\left\{c_{U v}(\alpha, \beta)+f_{v}(\beta)\right\}\right\} \\
\text { for } u=T-1, T-2 \ldots 0 \text { and } \alpha=1, \ldots P_{u} \\
\text { whe re } P_{0}=P_{T}=1 .
\end{gathered}
$$

Recursive relations (8) and (9) represent a ( $n-1)$-dimensional state variable dynamic programming relationship and can be represented by an acyclic network with vertices $\alpha=1,2, \ldots, P_{t}$ for each $t \varepsilon K+\{0\}$. In this acyclic network each vertex $\alpha \varepsilon\left\{1,2, \ldots P_{u}\right\}$ is connected to another vertex $\beta \in\left\{1, \ldots, P_{v}\right\} \quad v>u$ by a directed edge $(\alpha, \beta)$ with cost $c_{u v}(\alpha, \beta)$. An optimal solution to $P$ is given by $f_{0}(\alpha=1)$ and is represented by the shortest path between vertex $\alpha(=1)$ in period 0 and vertex $\beta(=1)$ in period $T$. Once the costs $c_{u, v}(\alpha, \beta)$ for all the directed edges in the acyclic network are computed, $f_{0}(\alpha=1)$ can be derived easily using a shortest route algorithm [3]. Since the total number of states at each stage $t$ is $n(R(t+1, T)+1)^{n-1}+1$, the number of computations per stage is of the order of $(R(1+t, T))^{(n-1)(n-1)}$. For $n=3$, the number of computations per stage is of the order of $(R(1+t, T))^{4}$ which is feasible on present large computers [8]. For $n>3$, it will be demonstrated in $\$ 4.4$ that when the demand functions can be assumed to be linear, considerable reduction on the number of $(n-1)$-dimensional state variables can be achieved, making recursive relations (8) and (9) still tractable when $n$ is not too large (e.g. for $n$ not more than 4 or 5 ).

For any value of $n$, however, for the procedure to be efficient, the subproblems for deriving $c_{u v}(\alpha, \beta)$ must be soluble easily. We shall now show that to be so for the case $n=2$. For the more general case where $n>2$, we shall also show that though the subproblems are slightly more complicated, they can still be solved efficiently by the approach indicated for $n=2$.

\subsection{Subproblems}

In the calculations in a subproblem to derive $c_{u, v}(\alpha, \beta)$ for $n=2$ three cases can be clearly distinguished, with $\alpha$ corresponding to the states of each of the three groups (as classified in \$3.1) falling under one case.

Case $(0)$ is the case where the $\alpha$ corresponds the state of group 0 . Here we have $I_{1 u}=I_{2 u}=0$. Since (7) is satisfied for periods $u+1$ to $v-1$, by Lemma 1 (properties (7a) and (7c)) we have: 


$$
y_{i j t}=x_{i t}=0 \text { for } i \neq j ; i, j \varepsilon J, \quad t \varepsilon\{u+2, \ldots, v\}
$$

This implies only $y_{i j t, u+1}$ and $x_{i u+1}$ for $i \neq j$; $i, j \varepsilon J$ can be nonzero. Hence, we now need to evaluate only the three remaining possibilities where there can be no cycle, i.e.

$$
\begin{array}{r}
\text { a. } x_{1 u+1}=R_{1}(u+1, v)+I_{1 v} ; \quad x_{2 u+1}=R_{2}(u+1, v)+I_{2 v} \\
\text { with } y_{12, u+1}=y_{21, u+1}=0 \\
\text { c. } x_{1 u+1}=R(u+1, v)+I_{1 v}+I_{2 v} ; \quad y_{12, u+1}=R_{2}(u+1, v)+I_{2 v} \\
\text { with } x_{2 u+1}=y_{21, u+1}=0 \\
x_{2 u+1}=R(u+1, v)+I_{1 v}+I_{2 v} ; \quad y_{21, u+1}=R_{1}(u+1, v)+I_{1 v} \\
\text { with } x_{1 u+1}=y_{12, u+1}=0 .
\end{array}
$$

Since $\beta$ specifies $I_{I v}$ (and $I_{2 v}$ ), in each of the three situations the value of the nonzero variables are specified uniquely and the costs are, therefore, trivially obtained. The minimum of the three costs is $c_{U V}(\alpha, \beta)$.

In case $(i) \alpha$ corresponds to a state of group 1 . Here we have $I_{2 u}>0$, $I_{I U}=0$. Again by Lemma 1 we have

$$
\begin{aligned}
& y_{12 t}=x_{2 t}=0 \text { for } t \varepsilon\{u+1, \ldots v\} \\
& y_{21 t}=x_{1 t}=0 \text { for } t \varepsilon\{u+2, \ldots v\} .
\end{aligned}
$$

But $x_{1 u+1} \cdot y_{21, u+1}=0$ by Lemma 1 (property (7b)).

Thus for extreme flow and feasibility we must have only one of two possibilities, i.e.

a. $\quad I_{2 u}=R_{2}(u+1, v)+I_{2 v}$ with $x_{1 u+1}=R_{1}(u+1, v)+I_{1 v} ; y_{21, u+1}=0$ or

b. $\quad I_{2 u}=R(u+1, v)+I_{2 v}+I_{1 v}$ with $y_{21, u+1}=R_{1}(u+1, v)+I_{I v}$;

$$
x_{1 u+1}=0 \text {. }
$$

In either case the values of the nonzero variables are uniquely specified. The minimum cost $\left(c_{U V}(\alpha, \beta)\right)$ can thus be obtained easily. It should be noted that when $I_{2 u}$ does not equal the two values specified, the flow is nonextreme and hence, in these cases $e_{u v}(\alpha, \beta)$ can be set arbitrarily high. Case ( $i i)$ is the case where $\alpha$ corresponds to a state of group 2 . This is similar to case (i) and need not be elaborated further.

For the more general case where $n>2$, the approach indicated can also be applied to derive $c_{U V}(\alpha, \beta)$ efficiently. For example, when $n=3$, we 
have four cases i.e. case (0) where $I_{I U}=I_{2 u}=I_{3 u}=0$, case (i) where $I_{I u}=0, I_{2 u}>0, I_{3 u}>0$, case (ii) where $I_{I u}>0, I_{2 u}=0, I_{3 u}>0$ and case (iii) where $I_{I u}>0, I_{2 u}>0, I_{3 u}=0$. For case (0) Lemma 1 implies only $y_{i j, u+1}$ and $x_{i u+1}$ can be nonzero. In this case we need to evaluate only 16 possibilities, ${ }^{2}$ each with the nonzero variables uniquely specified. For case $(i)$ the number of possibilities that need to be evaluated depends on whether $I_{2 u}$ (or $I_{3 u}$ ) is zero or positive. Assuming that $I_{1 u}=0, I_{2 u}>0$ and $I_{3 u}>0$, we now need to evaluate only three possibilities. ${ }^{3}$ However if $I_{I u}=0, I_{2 u}=0$ and $I_{3 u}>0$, we now need to evaluate seven possibilities, ${ }^{4}$ each with the nonzero variables uniquely specified. Cases ( $i$ ) and ( $i$ ii) are similar to case (i) and can be analysed in the same manner.

Having shown that $c_{u v}(\alpha, \beta)$ can be trivially derived, we are thus justified to say that recursive relations (8) and (9) provide an efficient algorithm for solving problem $P$ with $n$ not more than 3 .

\subsection{Remarks}

For $n>2$, recursive relations (8) and (9) are entirely different from recursive relations (17) and (18) of [2], since the latter cannot be extended to consider the general case of more than two producing regions. Even for the special case of $n=2$, although in the main problem recursive relations (9) and (9) are similar to recursive relations (17) and (18) of [2], an important difference exists between the two sets of relations in terms of the solution philosophy used and the computational efforts required for solving the

${ }^{2}$ The possibilities are (i) $x_{1 u+1}>0, x_{2 u+1}>0, x_{3 u+1}>0$, (ii) to (vii) with two of three capacity variables nonzero and one of three conversion variables nonzero (e.g. $x_{1 u+1}>0, x_{2 u+1}>0$ and $y_{13, u+1}>0$ ) and (viii) to (xvi) with one capacity variable and two conversion variables nonzero (e.g. $x_{1 u+1}>0 \quad y_{12, u+1}>0$ and $\left.y_{13, u+1}>0\right)$.

${ }^{3}$ The three possibilities are (i) $x_{1 u+1}>0$, (ii) $y_{21, u+1}>0$ and
(iii) $y_{31, u+1}>0$. (iii) $y_{31, u+1}>0$.

${ }^{4}$ The seven possibilities are (i) $x_{1 u+1}>0, x_{2 u+1}>0$, (ii) to (v) with either $x_{1 u+1}$ or $x_{2 u+1}$ nonzero and one conversion variable nonzero (e.g. $x_{1 u+1}>0$ and $y_{32, u+1}>0$ ) and (vi) to (vii) with no capacity variable nonzero but two conversion variables nonzero (e.g. $y_{31, u+1}>0$ and
$y_{32, u+1}>0$ ). 
subproblems. As demonstrated in $\$ 4.2$ for recursive relations (8) and (9) in the case where $n=2$, each $c_{U v}(\alpha, \beta)$ can be derived after merely three (case 0 ) or two (in case (i) or (ii)) sets of arithmetic operations. However, for recursive relations (17) and (18) of [2] each $d_{u, v}(\theta, \phi)$ can only be derived after $(v-(u+1))^{2}$ arithmetic operations (see $\$ 4.1$ of [2]). Hence, the computational efforts required to solve the subproblems in recursive relations (8) and (9) when $n=2$ is trivial and constant, whereas the computational efforts required to solve the subproblems in recursive relations of (17) and (18) of [2] is a square function of $v$ and $u$.

\subsection{Simplifications}

If the demand increments $r_{i t}$ are all even integers (including zero), we can as in [2] restrict the value of the state variable $\alpha$ in the main problem to those associated with only even values (including zero) of $I_{i t}$. The reader is referred to [2] for the proof.

Another important simplification arises when the demand for each type of equipment can be assumed to grow linearly, i.e. $r_{i t}=r_{i}$ for $i \varepsilon J, t \varepsilon K$. This simplification is presented as the following Lemma.

Lemma 2. For a problem $P$ where the demand grows linearly, i.e. $r_{i t}=r_{i}$ for $i \in J, t \in K$, at any period $t$ we can in the main problem restrict the value of $\alpha$ to those associated with the values of $I_{j t}$ for $j \in J_{i}$ (i.e. group $i$ states) which are partial sums of $r_{i}$ for $i \varepsilon J$ as follows :

$$
\text { Group } i: I_{j t}=\sum_{i \varepsilon J} a_{i} r_{i} \quad \begin{aligned}
& \text { for } j \varepsilon J_{i} \text { and } I_{i t}=0 \\
& \text { where } a_{i} \varepsilon\{0,1,2, \ldots T-t\} .
\end{aligned}
$$

Proof: Condition (10) follows from the concept of exact requirement introduced in [11]. This condition follows from the rationale that $I_{j t}>0$ with $I_{i t}=0$ implies that in an extreme flow of $G$ there must exist some subset of nodes $\left\{m_{\ell_{0} \tau_{0}}, m_{\ell_{1} \tau_{1}}, \ldots, m_{\ell_{k} \tau_{k}}\right\}$ where $\ell_{1}, \ldots, \ell_{k} \varepsilon J, \tau_{1}$, $\ldots, \tau_{k} \varepsilon\{t+1, \ldots, T\}$ and $\ell_{0}=j, \tau_{0}=t+1$ with a corresponding total demand that can be met exactly by idle capacity $I_{j t}$. If such a set does not exist, then it implies that there exists at least one node $m_{\ell \tau} \ell \varepsilon J$, $\tau \varepsilon\{t+1, \ldots, T\}$ whose associated demand is partially met from idle capacity $I_{j t}$ and partially from another capacity installation $x_{p q}, p \varepsilon J$, $q \varepsilon\{1,2, \ldots, \tau\}$ leading to the existence of a cycle of active arcs in $G$.

Lemma 2 effectively reduces the values of $\alpha$ in the main problem, at any period $t$, from $n(R(t+1, T)+1)^{n-1}+1$ to approximately $n(T-t)^{n(n-1)}$. 
Since at each stage $t$ the number of levels of $I_{i t}$ that needs to be enumerated is approximately $(T-t)^{n}$, this implies that the total number of distinct $(n-1)$-state variable is about $n(T-t)^{n(n-1)}$. This is an important reduction since it reduces the computational effort from one that is dependent on the demand parameters (i.e. $r_{i t}$ ) to one that is dependent on the planning horizon (i.e. $T$ ), which is typically a small number between 10 to 20 years. Hence when $T$ is about 10 years, the problem may be tractable on large computers when $n$ is not more than 4 or 5 . It should be noted that a simplification similar to Lemma 2 cannot be derived for the problem considered in [2].

A similar version of condition (10) can also be derived for problem $P$ in which $r_{i t}$ are arbitrary and non-negative. However, the number of distinct values which the partial sums of $r_{i t}$ for $i \varepsilon J$ and $t \varepsilon K$ can assume would be so many that it is, in this case, more efficient to enumerate the values of $I_{j t}$ for $j \varepsilon J_{i}$ (for states of group $i$ ) from 1 to $R(t+1, T)$ for $t \varepsilon K$.

\subsection{Conventional dynamic programming recursion}

Problem $P$ can also be solved using the conventional dynamic programming recursion with time periods as stages and excess capacities of the $n$ types of equipment as $n$-dimensional state variables. The computational effort in using this recursion, however, is much greater than recursive relations (8) and (9). The main computational saving in using recursions (8) and (9) is that the state dimensionality in the main problem has been reduced to $n-1$, with easy subproblems for the derivation of $c_{U v}(\alpha, \beta)$.

\section{A Numerical Example}

In this section, the application of recursive relations (8) and (9) is illustrated through solving a numerical example.

Consider a problem $P$ with $n=2$ and $T=3$ as represented by Figure 1, with $r_{i t}=2$ for $i=1,2$ and $t=1,2,3$.

The cost functions are assumed to be:

$$
C_{1 t}\left(x_{1 t}\right)=\left\{\begin{array}{lll}
0 & \text { if } & x_{1 t}=0 \\
(0.8)^{t-1}\left(4+5 x_{1 t}\right) & \text { if } & x_{1 t}>0
\end{array}\right.
$$




$$
\begin{aligned}
C_{2 t}\left(x_{2 t}\right) & = \begin{cases}0 & \text { if } x_{2 t}=0 \\
(0.8)^{t-1}\left(5+3 x_{2 t}\right) & \text { if } x_{2 t}>0\end{cases} \\
H_{12 t}\left(y_{12 t}\right) & = \begin{cases}0 & \text { if } y_{12 t}=0 \\
(0.8)^{t-1}\left(1+2 y_{12 t}\right) & \text { if } y_{12 t}>0\end{cases} \\
H_{21 t}\left(y_{21 t}\right) & = \begin{cases}0 & y_{21 t}=0 \\
(0.8)^{t-1}\left(2+3 y_{21 t}\right) & \text { if } y_{21 t}>0 .\end{cases}
\end{aligned}
$$

The vertices of the acyclic network representing the state variables $\alpha$ of the recursive relations (8) and (9) are given in Figure 2.

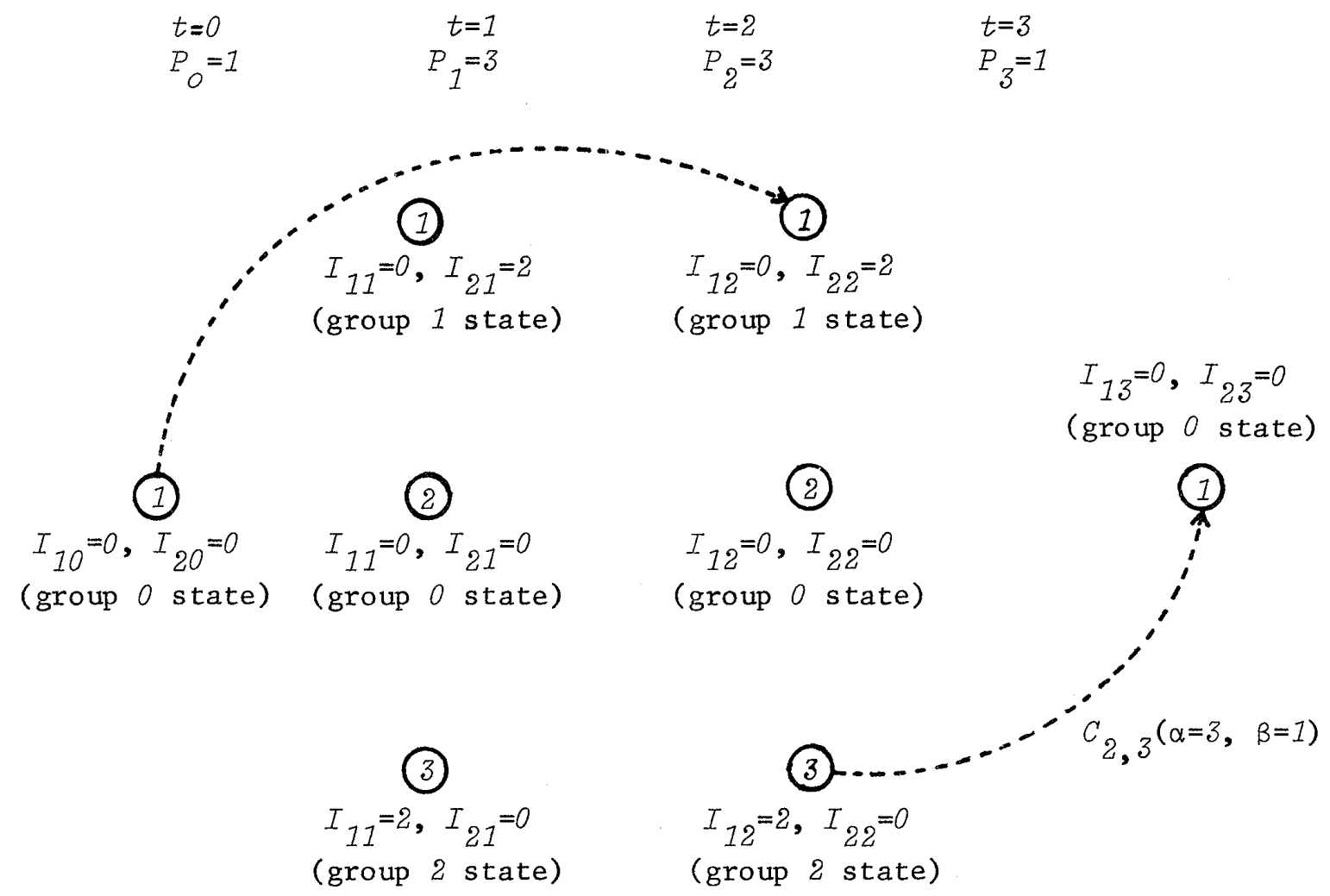

Vertices of Acyclic Network For Numerical Example

Figure 2 
It should be noted that since all the demand increments $r_{i t}$ are even integers, by the simplification of $\$ 4.4$, the state variables $\alpha$ in the acyclic network can be restricted to even values of $I_{i t}$. We shall now show the details for calculating $C_{u=0, v=2}(\alpha=1, \beta=1)$, i.e. the cost of the edge joining vertex 1 (i.e. $\alpha=1$ ) for $u=O\left(I_{10}=I_{20}=0\right)$ and vertex 1 (i.e. $\beta=1)$ for $v=2\left(I_{12}=0, I_{22}=2\right)$. It should be noted that $\alpha$ is in case (0) of \$4.2, where only $x_{i 1}$ and $y_{i j 1}$ for $i \neq j ; i, j \varepsilon J$ can be nonzero. Hence we need to evaluate only three possibilities, i.e.

a. $\quad x_{11}=R_{1}(1,2)+I_{12}=4+0=4$,

$x_{21}=R_{2}(1,2)+I_{22}=4+2=6$, with $y_{121}=0, y_{211}=0$

Cost for this case $=24+23=47$

b. $x_{11}=10, y_{121}=6$, with $x_{21}=0, y_{211}=0$

Cost for this case $=54+13=67$

c. $x_{21}=10, y_{211}=4$, with $x_{12}=0, y_{121}=0$

Cost for this case $=35+14=49$

Hence, $C_{0,2}(1,1)=47$ with $x_{11}=4, x_{21}=6$.

We sha11 now further show the details for calculating $C_{u=2, v=3}(\alpha=3$, $\beta=1) \quad i . e$. the edge joining vertex 3 for $u=2\left(I_{12}=2, I_{22}=0\right)$ and vertex 1 for $v=3\left(I_{13}=0, I_{23}=0\right)$. It should be noted that $\alpha$ is now in case (ii) of $\$ 4.2$ and we need to consider only one of two possibilities which are:

a. $I_{12}=R_{1}(3,3)+I_{13}$ with $x_{23}=R_{2}(3,3)+I_{23}$ and $y_{123}=0$

or

b. $\quad I_{12}=R(3,3)+I_{13}+I_{23}$ with $y_{123}=R_{2}(3,3)+I_{23}$ and $x_{23}=0$.

In this instance, $I_{12}=R_{1}(3,3)+I_{13}=2$. Hence $x_{23}=R_{2}(3,3)+I_{23}=2$ and $y_{123}=0$. Cost for this is $(0.8)^{2} \cdot 11=7.04$. Thus $C_{2,3}(3,1)=7.04$.

A11 the other edge costs in the acyclic network can similarly be calculated. These costs are given in Table 1.

The problem is then reduced to one of finding the shortest route through the acyclic network. It can easily be verified using a standard shortest route algorithm [3] that the least cost path from vertex 1 for $u=0$ to vertex 1 for $v=3$ is given by $C_{02}(1,1)+C_{23}(1,1)=47+8.96=55.96$, implying that the optimal solution is $x_{11}=4, x_{21}=6$ and $x_{13}=2$ with all other variables zero. 


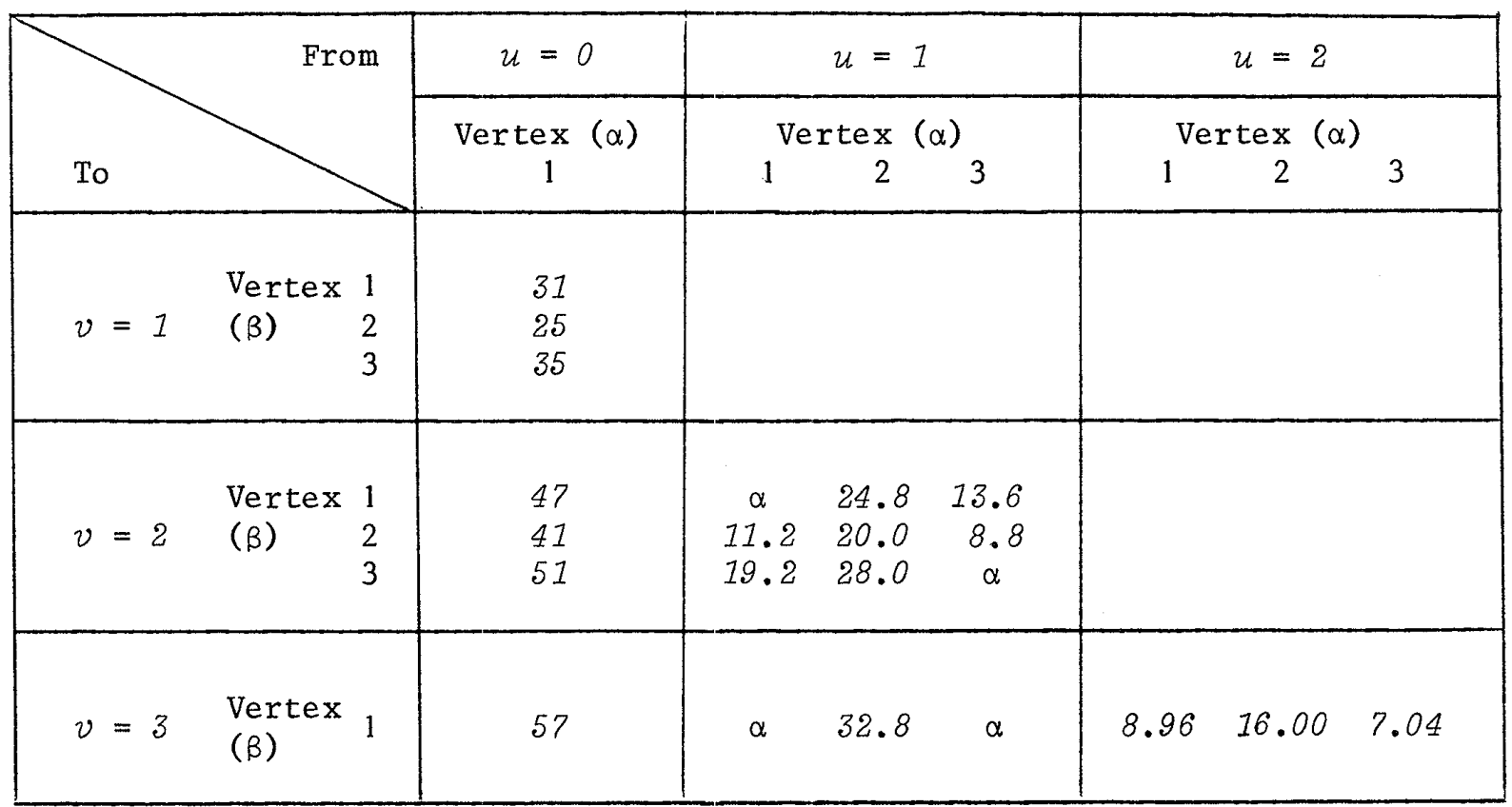

Note: Each entry denotes an edge cost e.g. $C_{u=1, v=2}(\alpha=2, \beta=3)=28.0$.

Edge Costs For Acyclic Network For Numerical Example

$\underline{\text { Table } 1}$

\section{Extensions to Problem $P$ with $n=2$}

In this section, we consider the various possible extensions to the model $P$. For brevity of presentation we shall discuss the case where $n=2$. The approaches used are, however, applicable for $n>2$.

\subsection{Idle capacity maintenance cost}

Maintenance cost of idle capacity in most realistic situations is negligible and has been ignored here. However it can, if necessary, be incorporated into model $P$ by defining the cost function $G_{i t}\left(I_{i t}\right)$ to represent the cost of maintaining $I_{i t}$ units of idle capacity of type $i$ equipment in period t. Function $G_{i t}(\cdot)$ is assumed to be concave, nonnegative and nondecreasing in the interval $[0, \alpha)$. Objective (1) would now include the terms $\sum_{i \varepsilon j} \sum_{t \varepsilon K} G_{i t}\left(I_{i t}\right)$. Assuming that the problem has a finite minimum, it can be solved by the approach discussed in $\S 3$ and $\S 4$. 


\subsection{Backlogging of equipment capacity}

In a situation where short-term leasing of equipment is available, then it may be realistic to incorporate the possibility of backlogging of each or both type of equipment capacity into the problem $P$. It needs to be pointed out that we assumed it is not possible to have an inventory or backlog of the service provided by the equipment. However, inventory of equipment capacity in the form of idle capacity and backlog of equipment capacity in the form of short-term leasing of capacity are now permitted. This can be achieved by defining: $I_{i t}=Q_{i t}-L_{i t}$, with $Q_{i t}, L_{i t} \geqslant 0$ for $i \varepsilon J, t \varepsilon K$ with the imposed condition that

$$
Q_{i t} \cdot L_{i t}=0 \text { for } i \varepsilon J, t \varepsilon K \text {. }
$$

The variables $Q_{i t}$ and $L_{i t}$ now represent the amount of idle and backlog capacity respectively of type $i$ equipment at period $t$. In terms of the network representation, this involves the addition of arcs $\left(m_{i t+1}, m_{i t}\right)$ to represent $L_{i t}$ with $Q_{i t}$ now represented by arcs $\left(m_{i t}, m_{i t+1}\right)$. We now define $F_{i t}\left(I_{i t}\right)$ as the cost of backlogging $L_{i t}$ units of type $i$ equipment capacity at period $t$, where $F_{i t}(\cdot)$ is again assumed to be concave, nonnegative and nondecreasing over the interval $[0, \alpha)$. Objection function (1) now would include the terms $\sum_{i \varepsilon J} \sum_{t \varepsilon K} F_{i t}\left(L_{i t}\right)$.

Since each node $m_{i t}$ now has four arcs representing $x_{i t}, y_{j i t}(j \neq i)$, $Q_{i t-1}$ and $L_{i t}$ directed into the node, Lemma 1 can be expanded as:

Lemma 3. Every extreme point of $P$ satisfies

$$
\begin{array}{ll}
x_{i t} \cdot Q_{i t-1}=0 & \text { for } i \varepsilon J, t \varepsilon K \\
x_{i t} \cdot L_{i t}=0 & \text { for } i \varepsilon J, t \varepsilon K \\
x_{i t} \cdot y_{j i t}=0 & \text { for } i \neq j ; i, j \varepsilon J, t \varepsilon K \\
y_{j i t} \cdot Q_{i t-1}=0 & \text { for } i \neq j ; i, j \varepsilon J, t \varepsilon K \\
y_{j i t} \cdot L_{i t}=0 & \text { for } i \neq j ; i, j \varepsilon J, t \varepsilon K \\
L_{i t} \cdot Q_{i t-1}=0 & \text { for } i \varepsilon J, t \varepsilon K .
\end{array}
$$

The proof of this Lemma is the same as the proof for Lemma 1 and needs no further elaboration.

At each period $t$, the number of states of groups 1 and 2 (as classified in \$3.1) would now have to be expanded to include negative values of $I_{i t}$, i.e. 


$$
\begin{aligned}
& \text { Group 1: } I_{2 t} \neq 0, I_{1 t}=0 \\
& \text { Group 2: } I_{2 t}=0, I_{1 t} \neq 0 .
\end{aligned}
$$

Now states in each groups 1 and 2 can assume a maximum of $R(1, T)$ levels. Hence at each period $t$, we have to enumerate $2 R(1, T)+1$ levels. The recursive relations $(8)$ and $(9)$ is still applicable except that now $P_{t}=2 R(1, T)+1$ for $t \in K-\{T\}$ with $P_{O}=P_{T}=1$.

In using recursive relations (8) and (9) the subproblems for calculating $c_{u v}(\alpha, \beta)$ even for $n=2$ is now slightly more complex. Referring to the classification of $\$ 4.2$, in case ( 0 ) we can have four subcases:

$$
\begin{aligned}
& Q_{1 t}>0, Q_{2 t}>0, L_{1 t}=I_{2 t}=0 \text { for } t=u+1, \ldots, v-1 \\
& Q_{1 t}>0, L_{2 t}>0, L_{1 t}=Q_{2 t}=0 \text { for } t=u+1, \ldots, v-1 \\
& L_{1 t}>0, Q_{2 t}>0, Q_{1 t}=I_{2 t}=0 \text { for } t=u+1, \ldots, v-1 \\
& L_{1 t}>0, L_{2 t}>0, Q_{1 t}=Q_{2 t}=0 \text { for } t=u+1, \ldots, v-1 .
\end{aligned}
$$

As shown in $\$ 4.2$ for (13a) we have only three possibilities in which we need to compute the costs. For (13b), it can easily be verified by Lemma 3 (using properties (12d) and (12e)) that $y_{21 t}=0$ for $t=u+2, \ldots, v$ and $y_{12 t}=0$ for $t=u+1, \ldots, v-1$. Hence we have only five possibilities in which the costs need to be computed. The five possibilities are:
(a) $x_{1 u+1}>0$ with $y_{12 v}>0$,
(b) $x_{1 u+1}>0$ with $y_{2 v}>0$,
(c) $x_{1 u+1}>0$,
(d) $x_{2 v}>0$ with $y_{21, u+1}>0$ and
(e) $y_{21, u+1}>0$.

Again in each of the five situations the nonzero variables are uniquely specified and the cost can be derived trivially. Conditions (13c) and (13d) are similar to $(13 b)$ and (13a) respectively. Hence for case $(0)$, we have to derive 16 cost data, the minimum of these being $c_{u v}(\alpha, \beta)$.

In case ( $i$ ) we also have the four subcases (13a) to (13d). For (13a) it can be shown that, since now $I_{2 u}$ can be negative, we now need to compute the costs for five possibilities. ${ }^{5}$ In each of (13b), (13c), (13d), we have eight possibilities. ${ }^{6}$ Hence we compute $c_{u v}(\alpha, \beta)$ with $\alpha$ from a group 1 state we have to derive 29 cost data and select the minimum.

Case ( $i i$ ) is similar to case (i) and will not be further elaborated. We have now shown that the subproblems can still be solved easily for the 
backlogging case, justifying the fact that recursive relations (8) and (9) can still be applied efficiently.

\subsection{Initial capacities}

Initial capacity in either or both type of equipment can also be incorporated into model $P$. In this case, the state specified by $\alpha$ at stage 0 would correspond to the capacity status of each type of equipment. Recursive relations ( 8 ) and (9) can still be used to solve the problem. However now the network representing $G$ is no longer a single-source network and Lemma 1 need not necessarily hold true. Now the subproblem for calculating $c_{u v}(\alpha, \beta)$ is slightly more complicated. Referring to the classification of $\$ 4.2$, case $(0)$ remains unchanged since $I_{I u}=I_{2 u}=0$ implies that Lemma 1 still holds for nodes $m_{i \tau}$, $i \in J$ and $u<\tau \leqslant v$. In case $(i)$, we still can have at most one installation for each type of equipment between periods $u+1$ and $v$, i.e. we can have no installation, only one installation, or two installations with one installation per type of equipment. The cost for the possibility of no installation between $u+1$ and $v$ is uniquely specified, since for feasibility $y_{21, u+1}$ must be nonzero and, hence, $y_{21 t}$ for $t=u+2, \ldots, v$ and $y_{12 t}$ for $t=u+1, \ldots, v$ must be zero. Consider the possibility in which there is only one instazlation. When the expansion occurs at node $m_{2 \tau}$ for any $\tau$ between $u+1$ and $v$ or $m_{1 \tau}$ for any $\tau$ between $u+2$ and $v$ the cost is uniquely specified since the value of the expansion is known and $y_{21, u+1}>0$ for feasibility. There are, therefore, $(v-u)+(v-u-1)$ such

\footnotetext{
${ }^{5}$ The possibilities are (a) $x_{1 u+1}>0$ with $y_{12, u+1}>0$, (b) $x_{1 u+1}>0$ with $x_{2 u+1}>0$, and (c) $x_{1 u+1}>0$, (d) $x_{2 u+1}>0$ with $y_{21, u+1}>0$, and (e) $y_{21, u+1}>0$.
}

${ }^{6}$ For ( $13 \mathrm{~b}$ ) the possibilities are (a) no positive installation or conversion variable between periods $u+1$ and $v$, (b) $y_{12, u+1}>0$, (c) $y_{21, v}>0$ (d) $x_{2 u+1}>0$, (e) $x_{2, u+1}>0, y_{21, v}>0$, (f) $x_{2 u+1}>0, x_{1 v}>0$, (g) $x_{1 v}>0$, (h) $x_{1 v}>0, y_{12, u+1}>0$. For (13c) and (13d) the possibilities are similar and, therefore, need not be given here. evaluations. When the expansion occurs at node $m_{1 u+1}$ we have $2(v-u)$ possibilities, each with $y_{21 \mathrm{\tau}}$ or $y_{12 \tau}$ (but not both) nonzero for $\tau \varepsilon\{u+1, \ldots, v\}$. Hence we have a total of $4(v-u)-1$ possibilities to consider when there is only one expansion. When there are two installations between periods $u+1$ and $v$, there cannot be any capacity conversion 
between periods $u+1$ and $v$. This implies we now have $u-v$ possibilities with $x_{1 u+1}>0$ and $x_{2 \tau}>0$ for any $\tau$ between $u+1$ and $v$. Hence in this case (and also case $(i i)$ ), we have to consider $5(v-u)$ possibilities, each with the cost uniquely specified.

\subsection{Arbitrary demand}

The case in which the demands are arbitrary (i.e. not necessary nondecreasing) may be realistic in some situations. For example, some public railway administrations have experienced an absolute decline in demand for passenger traffic (cars) due to increasing competition of other modes of passenger transportation, while the demand for freight cars have generally maintained a steady growth. The formulation and network representation of problem $P$ still remains valid when the demands are arbitrary. Now we have $R_{i}(1, t) \geqslant 0$ for $i \varepsilon J$ and $t \varepsilon K$, but $r_{i t}$ is unrestricted in sign. In the network each $r_{i t}<0$ would be represented by an inflow of $r_{i t}$ units into node $m_{i t}$. As in the case with initial capacities, the network is now a multi-source network and Lemma 1 is no longer valid. Recursive relations (8) and (9) can still be used to solve the problem, but the subproblems would have to be solved in the manner described in $\$ 6.3$.

\section{Conclusions}

In this paper, a recursive relationship is developed to solve the problem of capacity installation for several types of related equipment with conversion possibility from one type to the other - a problem encountered particularly in the transport and manufacturing industries.

This recursive relation is an extension and refinement of the recursive relation developed in [2], and is quite efficient when the types of equipment is not too large (e.g. $\leqslant 4$ ). This is in contrast to the recursive relations in [2] which is limited to the case of two producing regions only. Even when the types of equipment available is two (i.e. $n=2$ ), it is further shown that the solution philosophy used to solve the subproblems is quite different, and more efficient, than that used in [2]. Important implications and extensions to the basic problem considered are also derived. 


\section{References}

[1] Dantzig, G.B.: Linear Programming and Extensions. Princeton University Press, Princeton, New Jersey, 1963.

[2] Fong, C.o. and Rao, M.R.: Capacity Expansion with Two Producing Regions and Concave Costs. Management Science, Vo1. 22 (1975), pp. 331-339.

[3] Ford, L.R., Jr. and Fulkerson, D.R.: Flows in Networks. Princeton University Press, Princeton, 1962.

[4] Hadley, G.: NonZinear and Dynamic Programming. Addison-Wesley Publishing Company, Reading, Massachusetts, 1964.

[5] Johnson, E.L.: Networks and Basic Solutions. Operations Research, Vo1. 14 (1966), pp. 619-623.

[6] Kalotay, A.J.: Capacity Expansion and Specialisation. Management Science, Vo1. 20 (1973), pp. 56-64.

[7] Manne, A.S. and Vernott, A.F., Jr.: Optimal Plant Size with Arbitrary Increasing Time Paths of Demand: A.S. Manne (ed.), Investment for Capacity Expansion: Size, Location and Time Phasing. M.I.T. Press, Cambridge, Massachusetts, 1967.

[8] Petersen, E.R.: A Dynamic Programming Model for the Expansion of Electrical Power Systems. Management science, Vo1. 20 (1973), pp. 656-664.

[9] Sengupta, J.K. and Fox, K.A.: Optimization Techniques in Quantitative Economic Models. North-Holland, Ams terdam, 1969.

[10] Wagner, H.M. and Whitin, T.M.: Dynamic Version of the Economic Lot Size Mode1. Management Science, Vo1. 4 (1958), pp. 89-96.

[11] Zangwil1, W.I.: A Deterministic Multi-Period Production Scheduling Mode1 with Backlogging. Management Science, Vo1. 13 (1966), pp. 105-119.

[12] Zangwil1, W.I.: A Backlogging Model and a Multi-Echelon Model of a Dynamic Economic Lot Size Production System - A Network Approach. Management Science, Vo1. 14 (1969), pp. 506-527.

Chan Onn FONG: Faculty of Economics and Administration, University of Malaya, Kuala Lumpur, Malaysia. 